\title{
Semantic Processes Modelling Independent of Manufacturing Infrastructures
}

\author{
Antonio Ferrándiz-Colmeiro, Virgilio Gilart-Iglesias, Francisco Maciá-Pérez \\ Computer Science Department \\ University of Alicante \\ \{aferrandiz, vgilart, pmacia\}@dtic.ua.es
}

\begin{abstract}
This paper presents a proposal enabling the modelling of manufacturing processes independently of the structure and composition of the industrial plant where these processes will ultimately be implemented. The rationale for the proposal is in the incorporation of knowledge, supported by ontologies regarding manufacturing processes, industrial machinery and the industrial plants involved. With this knowledge, process engineers can focus their efforts exclusively to define the activities to be performed on the raw material (abstract model), allowing the system to infer what other activities should be incorporated in the abstract model to obtain a particular manufacturing process tailored to a particular industrial plant. To refine the proposal, a case study is put forward in which the management system was able to infer the way for the raw material to be processed according to the abstract model and automatically composed the activities necessary for achieving it. Finally, an implementation of this management system for processes is presented together with the design of two models of industrial plant that demonstrate the validity of our proposal.
\end{abstract}

\section{Introduction}

The capacity that the Internet has provided to customers to select the consumer articles that best suit their needs at any given time and at the best prices is causing manufacturing organisations to move from traditional production paradigms centred on mass production to models that facilitate mass customization [1]. This new scenario demands that organisations implement new, more dynamic and more flexible management models that easily enable them to adapt to environmental changes brought about by market demand [2][3].

In order to respond to the needs of new models, the BPM (Business Process Management) paradigm has become the focus of more appropriate business process management [3] because it contemplates continuous change as one of its main characteristics in addition to the dynamic adaptation of the business processes and IT infrastructures that sustain them.
Within the framework of manufacturing organisations it is difficult to completely implement BPMSs due to the conceptual and technological gap between the enterprise level and productions levels, caused by the rigidity of the elements located in the lower levels of production [4]. Offering a vision of industrial machinery as a service (IMaaS) [5], integrated in accordance with a ServiceOriented Architecture (SOA) paradigm, has led to a successful proposal for solving this problem by considering industrial machinery manufacturing processes as just another element of the business process management system.

On removing this gap, a whole range of possibilities for process management in manufacturing organisations comes into play. However, this range of possibilities is shifting the bottleneck that traditionally existed between the company and the plant levels to the modelling domain and process management.

In order to mitigate the burden of this shift, the present article proposes a model that automates modelling and composition of processes in line with the organisation's strategic objectives and their subsequent deployment and execution in various manufacturing organisations independently of their physical structure. It adapts these processes in an agile way to the resources that exist in each of the organisations. To this end, the proposal is based on the synergy between the IMaaS model, which considers industrial machinery as a service [5], and the incorporation of knowledge in the definition of the processes and services involved through the use of ontologies described in [6].

The remainder of this document has been structured in line with this objective as follows. Section 2 briefly reviews the previous work on which this present proposal is based, placing special emphasis on the concept of IMaaS and reviewing the state of the art on related aspects. Next, the general proposal is presented, describing the model that makes the modelling of processes independent of the physical structure of the manufacturing organisation in which they are to be implemented. Section 4 describes the case study, continuing with the implementation of a prototype of the proposed system together with a testing scenario consisting of a set of industrial plant models. These are used to demonstrate the validity of the proposal and its 
viability in a real environment. Finally, the main conclusions from the research are presented along with lines of future work.

\section{Background}

The full integration of manufacturing processes into the global map of the business model has propitiated diverse solutions that have developed from traditional automation models integrated with the management levels by means of ad-hoc adapters to systems based on information technology applications that are currently achieving great success within the scope of integration, such as the transparent factory proposal [7], the use of the SOAP protocol as proposed by ABB [8] and systems based on Web Services [9].

There are more recent proposals, some of which are within the scope of European projects based on the use of Web Services and WS-*, that show the functionality of the production elements [10][4]. Other proposals, such as IMaaS, which is described in [4][5], present industrial machinery as part of a business process management system (BPMS), indicating its functionality as services in accordance with the SOA paradigms[11][5]. This latter focus is one that has been used in the general scenario described in the proposal of this paper in order to enable the implementation of BPMS in manufacturing organisations.

The IMaaS proposal removes technological and conceptual restrictions preventing the integration of business and manufacturing processes through the definition of two procedures. Firstly, the procedure of conceptual normalisation of industrial machinery establishes a methodology for removing the conceptual restrictions in a systematic way. This procedure translates manufacturing processes to the business domain and includes in industrial machinery all the elements necessary to visualise it as a process management system based on the BPM lifecycle model, obtaining industrial machinery as a BPM as a result. Secondly, the procedure for technological normalisation of industrial machinery establishes the technological architecture to support the model obtained in the previous procedure, displaying industrial machinery functions as services (IMaaS architecture). This removes traditional technological restrictions and brings flexibility and dynamism to the machinery [5].

In order to cover requirements such as selforganisation, automation and reactive and proactive behaviour, it is necessary for there to be communication between the organisation, the applications and the machinery. To enable this communication to exist it is essential to formally classify the information referring to a domain, for which ontologies are presented as useful tools. This information is interpretable by software agents in such a way that the interoperability of IT systems is enabled and the integration of the different systems within the company is strengthened, thus making it possible to establish reasoning, based on the internal logic of the ontology, that allows the different elements to automatically interact [12].

Various systems based on ontologies can be identified over the domain of manufacturing companies. As a sample of the conceptualisation of the domain, the MASON ontology [13] is used for the automatic reconfiguration of industrial machinery depending on the objective is shown in [14]. Within manufacturing systems based on services, there are a series of proposals focussed on representing the description of the services offered by machines in a semantic way. The utility of using Semantic Web Services for automation of production systems is described in [15].

However, manufacturing cannot be considered to be an isolated process within the company, rather it must be understood to be just another process within the company's business processes. In this field there is also a wide range of proposals. In [16], an architecture based on the semantic description of services and on a BPEL (Business Process Execution Language) engine is proposed as the means for executing said services. In [17] a comparison is made between BPEL and OWL-S (Ontology Web Language Service) and the conclusion is that OWL-S is more appropriate for dynamic environments and BPEL is the ideal choice for controlled workflows.

In the field of business process management, a set of limitations of BPM methodologies are identified in [18] and a proposal put forward for overcoming them by the combination of BPM and Semantic Web Services (SWS) to arrive at what the authors called Semantic Business Process Management.

A method for the integration of industrial machinery in BPM systems is presented in [6] in such a way as to achieve a higher degree of automation in the composition and in the deployment of the modelled processes, which are implemented as Web Services.

From the above analysis, it seems that BPM systems are the most appropriate for agile process management and the IMaaS approach has been presented as the solution facilitating the implementation of BPMs in manufacturing organisations as these enable overcoming technological and conceptual barriers between the business and manufacturing levels. In addition, the use of ontologies supporting manufacturing process management helps to increase the degree of automation over that of BPM systems alone when working in a particular domain. In this paper, the capabilities given by ontologies to help separate the process modelling effort from the manufacturing environment are exploited, enabling greater agility when composing processes and deploying them in different manufacturing environments. 


\section{General Proposal}

The work described in this paper is focussed on a proposal for a model of integratal management of business and manufacturing processes carried out in manufacturing organisations. The proposal automates the modelling of processes in accordance with the organisation's strategic objectives and their subsequent deployment and execution in a transparent way in any manufacturing environment, irrespective of the physical structure of its production elements.

Our proposal enables focussing on process definition without the need to consider the underlying technology. It encourages process engineers to generate optimum process structures for a specific production sector complying with legal regulations and good business and industrial practices. This facilitates reuse of the modelled processes and enables the establishment of generic process templates that can be deployed in any environment at any time.

\subsection{Conceptual Model}

The conceptual model is divided into three conceptual levels: business level, knowledge level and manufacturing level (Figure 1).

The business level is formed by the functionalities defined in the BPM lifecycle for process management, through which the Process Manager manages the business processes of the organisation, including the manufacturing processes as just another set of business process. This level includes extra functionalities enabling automation of the included functionalities starting from the knowledge provided by the lower levels of the model.

The features of the manufacturing processes are dependent on the manufacturing level, so a second intermediate level has been defined between the business level and the manufacturing level with the aim of making both domains independent through the introduction of knowledge in description of the processes and the production elements. This level has been denominated the knowledge level and includes conceptual information about the physical structure of the organisation's manufacturing elements and their technological features; the information about the processes that are carried out in the organisation and their relationship to the machinery responsible for their execution; and the services that expose the functionality of these processes. In this way, a view of industrial machines from a functional point of view is offered, at levels that are higher than treating them as a black box.

To achieve this abstraction, a conceptual and technological normalisation of industrial machinery at the level of the plant is necessary. This removes the gap in the organisation between the elements located at levels below that of production and the management elements at the level of the company. This normalisation is carried out in the manufacturing level. Thus, management can consider different industrial plants in the same way, irrespective of the mechanics of each industrial plant.

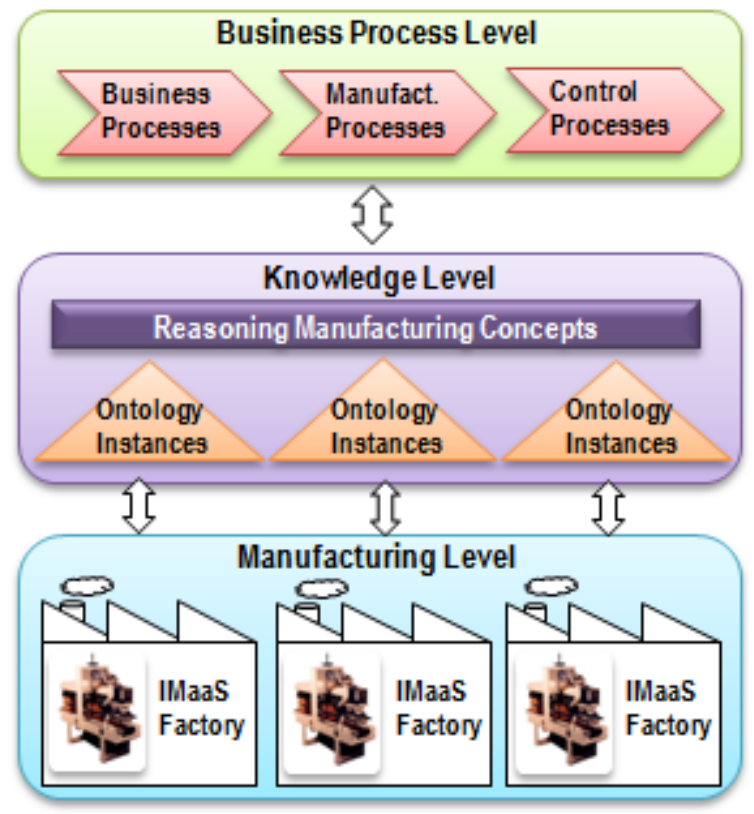

Figure 1. Conceptual proposal of the Manufacturing System independent of the plant level.

\subsection{Proposal architecture}

To implement the proposal described above, an SOA architecture has been specified based on the Matchmaker framework composed of: the IMaaS production system (manufacturing level); and the information system and process management system (business and knowledge levels); as shown in Fig. 2, where the IMaaS production system acts in the role of service provider, the information system acts as intermediary for service localisation and discovery and the process management system acts as IMaas service consumer.

\subsubsection{IMaas Production System}

The production system is composed in turn by a set of industrial machines offering functionality as business processes expressed as services (IMaaS). These processes are responsible for carrying out physical processes in the production environment. Each of these machines offers processes that can be performed as a service. These services are registered in the information system in such a way that they can be made available to be consumed. Also, each production system contains semantic information about the complete set of machines and processes that are available. Such semantic information corresponds to the instantiation of the production system elements (ABox) within general ontology proposed in [6] and described in the next section of this document. 


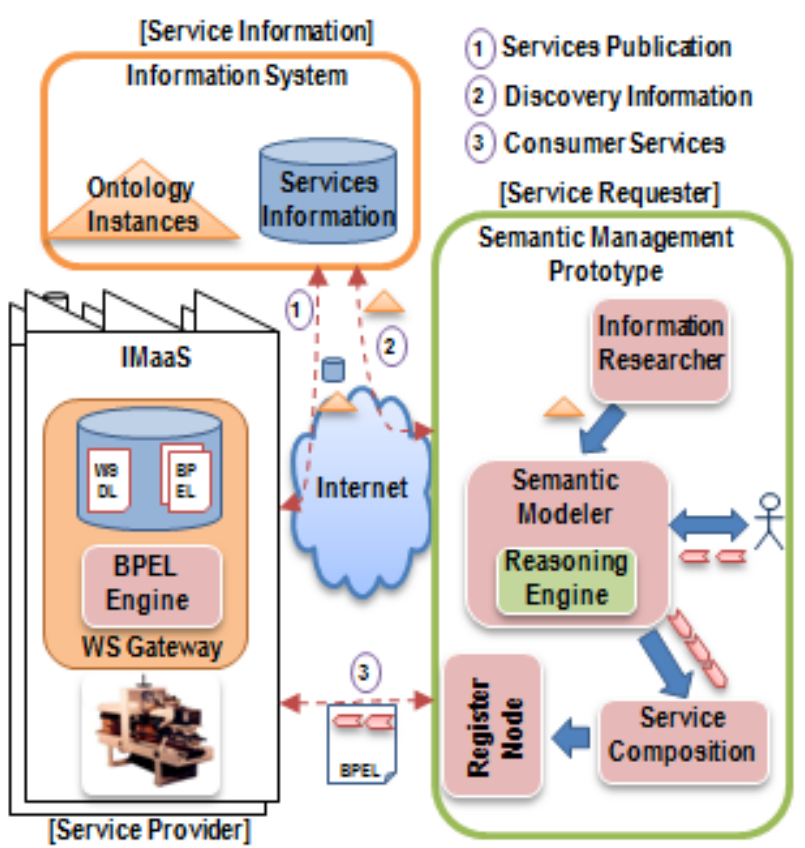

Figure 2. Proposal for the Manufacturing System distributed in various plants based on SOA.

\subsubsection{Information System}

The information system is composed of the description of the services available in the various manufacturing systems and the semantic knowledge base. The latter is formalised as an ontology (TBox) that contains a classification of the various machines that can be found in a manufacturing plant, the relationships between the various types of machines, what services are offered by each machine and the way in which each service is related to a set of generic manufacturing processes.

\subsubsection{Process Management System}

The process management system is responsible for the management of policies, objectives and business strategies using a BPMS system directed by semantic information. This system is composed of a business process modelling system that, starting from a functional process, automatically composes the complete process that reflects the specific characteristics of a particular manufacturing plant. A functional process is understood to be specification of the process to be performed, independently of how it should be performed. Once the modelled process is available, it is passed to a model compiler (Service composition), which, from the information specified in each ontology of each plant, together with the automatically composed process, is able to translate the model into an executable language that can be interpreted by the industrial machinery and physically carried out. This enables reducing the difficult communication between stakeholders of different areas, and who often do not manipulate the same concepts, by allowing the system to compose the services automatically.

The executable process obtained is sent to the manufacturing plant, in which it is registered and is instantiated in the ontology. In this way, there is feedback from the manufacturing system allowing the production plant to offer its new process as a service for future uses.

Because the proposal is based on distributed architecture and the composition system is based on a generic knowledge source in the ontologies, this system can be extended as shown in Figure 2, so that a single management system can operate various manufacturing plants remotely.

The management system allows storage of the functional processes so that, when the manufacturing plant changes or new manufacturing plants are incorporated into the company, the modelling and deployment of the already available processes to other plants is immediate. In turn, the system can manage the new processes generated by the new plant in an agile way. Thus, on the one hand, remodelling of manufacturing processes after changes at plant level can be avoided, and on the other hand, reuse of the modelled processes for deployment in different production environments is encouraged.

\section{Case Study}

To illustrate the proposal described above, a manufacturing system is presented in which only the processing (operations for the transformation of material) that must be performed on the raw material is modelled and it is the system that is responsible for orchestrating and composing the final process. It automatically calculates the route that the raw material must follow through manufacturing and incorporates the auxiliary processes necessary so that the machinery moves the raw material along this route. This allows the process engineer to model processes as a function of the established policies and business objectives at the company level.

The proposed ontology assembles the information referring to various types of machines existing at plant level together with their characteristics (such as: position, number of sensors, types of tools, etc.); each machine has a series of associated services, which implement manufacturing processes; and a hierarchical classification of the various manufacturing processes is defined. Figure 3 shows a simplified structure of the proposed ontology, which consists of three main classes: service, process and machine, and the semantic relationships between these classes or concepts. 


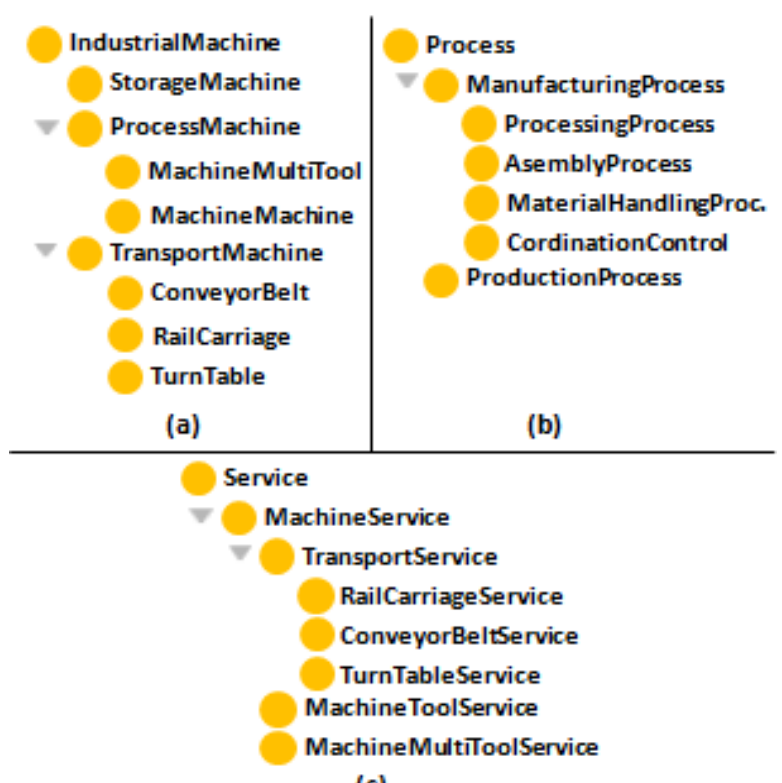

(c)

Figure 3. Implemented Ontology.

This ontology has also been extended using the language of logical rules, defining a series of rules that enable establishing relationships and generalisations between different concepts. Specifically, these rules have been used to establish the connections (physical pathways), either direct or indirect, between the various machines of a particular manufacturing plant. Thus, if a machine is connected through its $\mathrm{X}$ axis or its $\mathrm{Y}$ axis to another machine and this in turn is connected to a third machine, then there exists at least one connection between the first machine and the third. As will be seen in the composition of processes, these rules allow rapid calculation of the possible connected machines. This reasoning is performed on the ontology, allowing the separation of business strategies from the implementation of a particular software tool.

Starting from the functional process defined generically by the process engineer and independently of the infrastructure of the factory where it will be deployed, the system automatically composes the final process that is dependent on the infrastructure of the factory.

To do this, the system extracts information about the types of processes and their execution sequence.

Then, reasoning is performed on the ontology to obtain which machines carry out these processes using which services. From the information obtained about the machines, a search is made for all the possible routes through which the given final process can be composed (Figure 4).

Finally, the process modelled at an abstract level is translated into an executable format using the ontologies defined for each plant (ABox), establishing the relationship between the modelled processes and the services that will implement these processes.

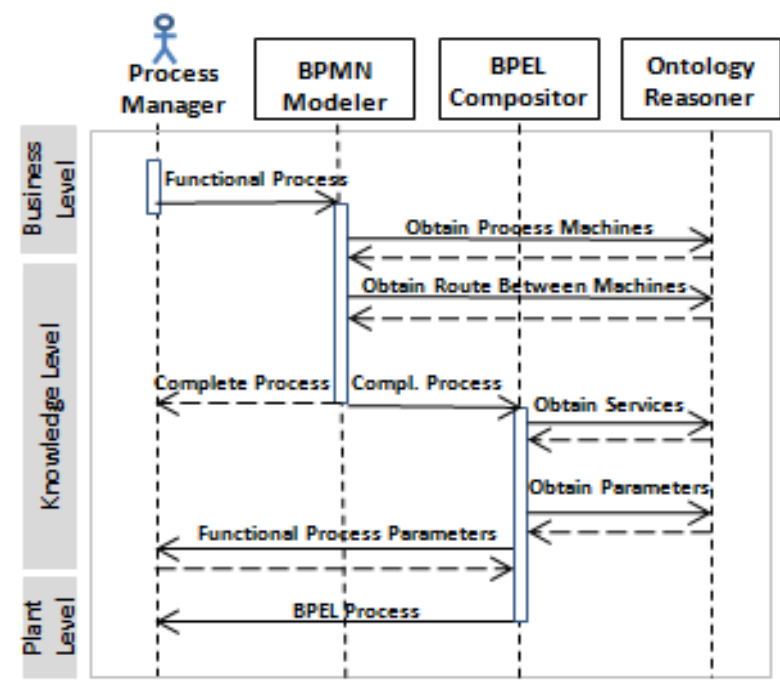

Figure 4. Sequence Diagram.

\section{Implementation and validation}

In order to validate the proposal, a set of experiments were designed on the implementation of a prototype based on the scenario proposed in figure 2. These experiments are summarised as follows: modelling a process with a limited set of manufacturing operations on a specific raw material; designing two different industrial plants but each capable of carrying out these operations (but with different machinery and different physical structure); deploying the abstract process with the operations on both factories; and finally, verifying correct execution.

\subsection{Implementation of the architecture}

The information system (IS) comprises all the elements and resources used by the other systems of the organisation to carry out the business or company activities. Among its included elements is the UDDI registry, implemented with the jUDDI v 2.0 server, that stores the information of the WSDL and BPEL documents describing the business functionalities and processes of the organisation and enables dynamic localisation of the services offered by the machinery and their decoupled consumption. The IS also contains the concepts ontology (TBox) implemented using the OWLDL (Ontology Web Language Description Logic) language and extended with SWRL (Semantic Web Rule Language) rules.

To implement the IMaaS manufacturing system, two industrial plants were designed with different features, both physical and technological, in order to validate the functionality of the proposal presented. Both factories were implemented for the company STAUDINGER GMHB, in accordance with the specifications defined by the research group. These factories were composed of a series of industrial machines to scale, which were 
controlled through the DCOM protocol by the ICPDAS I-7055D controller that works over the RS-485 protocol.

In the case of factory 1 (Figure $5 \mathrm{a}$ ), the control of the industrial machinery was carried out by the XPort embedded device. The device was adapted to work with the RS-485 protocol and acts as middleware between the manufacturing elements and the manufacturing process management system. A SOAP engine was implemented in this device for devices of limited resources using a web server in the embedded device. This allows offering the processes and activities provided by the machinery as Web Services (Table 1). A small BPEL engine was implemented on the SOAP engine to be capable of orchestrating and executing the services on the industrial machinery. Other Service Orientated Architecture (SOA) functionalities were also implemented such as, for example, the dynamic publication of services in the UDDI v 2.0 registry.

Table 1. Processes offered by the industrial machinery involved in the experiment.

\begin{tabular}{lll}
\hline \hline \multicolumn{1}{c}{ Name } & \multicolumn{1}{c}{ Arguments } & \multicolumn{1}{c}{ Description } \\
\hline \hline Conveyor Belt (CB) & \\
\hline \hline $\begin{array}{l}\text { MoveTo } \\
\text { Sensor }\end{array}$ & $\begin{array}{l}\text { Direction:INT }\{1,-1\} \\
\text { Sensor:INT }\{0-\mathrm{N}\} \\
\text { Stop:BOOL }\end{array}$ & $\begin{array}{l}\text { Move the piece to } \\
\text { until indicated } \\
\text { sensor. }\end{array}$ \\
\hline MoveToEnd & Direction:INT $\{1,-1\}$ & $\begin{array}{l}\text { Move the piece to } \\
\text { the end of the belt. }\end{array}$ \\
\hline \hline Turntable:Conveyor Belt (TT) & \\
\hline \hline TurnMove & Direction:INT $\{1,-1\}$ & $\begin{array}{l}\text { Turn the table and } \\
\text { expedite the piece }\end{array}$ \\
\hline \hline Rail Carriage:Conveyor Belt (RC) & \\
\hline \hline Transport & $\begin{array}{l}\text { Direction:INT }\{1,-1\} \\
\text { InputDirection:INT } \\
\text { OutputDirection:INT }\end{array}$ & $\begin{array}{l}\text { Get a piece, } \\
\text { transport and } \\
\text { expedite it. }\end{array}$ \\
\hline \hline Machine Tool (MT) & \\
\hline \hline Process & Time:INT & $\begin{array}{l}\text { Move the tool out } \\
\text { and down, process } \\
\text { the piece and move } \\
\text { the tool up and in. }\end{array}$ \\
\hline \hline & Time:INT & $\begin{array}{l}\text { Process the piece } \\
\text { with the indicated } \\
\text { tool. }\end{array}$ \\
\hline \hline Machine Multi Tool:Machine Tool (MMT) \\
\hline \hline
\end{tabular}

In the case of industrial plant 2 (Figure 5b), control was carried out with another type of embedded device as middleware, in this case based on a MOXA device offering some advantages over XPORT such as: more memory and higher computation capacity enabling, for example, the full implementation of the SOA architecture. Thus, two completely different factories, in terms of physical organisation and devices, were available. The Service Compositor also used the reasoning performed with PALLET to extract the information about the services that are implemented by particular processes.

\subsection{Experiment design}

Starting from the functional process diagram, the tests consisted in automatically obtaining two processes adjusted to the features of the industrial plants (Fig. 5a and $5 \mathrm{~b}$ ) using the reasoning performed on the ontology, which contained the information about these plants.

More specifically, a diagram in BPMN notation, representing a functional process to implement in any of the available factories, was introduced into the modelling tool for subsequent automatic composition of the process from the complete model for each chosen factory in order to deploy the process. Once the complete process model was available, automatic discovery of services was performed and the process translated into an executable language, BPEL, trying to minimise the interaction with the user during the translation, so that the user was only concerned in providing the service parameters involved in the functional process.

The functional process designed for the experiment was that shown in figure $6 \mathrm{a}$. The process comprised three sub-processes executed sequentially: drilling, polishing and painting. For simplicity, when these subprocesses have been performed on the material, the process was considered finished. However, in a real environment, the process would finish when the material is removed from the processing chain.

\subsection{Analysis of the results}

In plant 1 (Figure 5a), the machinery performing the drilling process is identified as MT2, the machine performing the polishing process is identified as MT1 and the machine performing the painting process is identified as MT3.

With the information contained in the functional diagram, the composition of the complete process was performed using the ontology containing the instantiation of plant 1 . A series of reasoning operations was performed on the ontology, which enabled knowing which services implement the specified processes, which machines offer these services and what possible routes can be established between these machines. The result was a complete process composed of a total of $18 \mathrm{sub}$ processes, of which 3 were functional and 15 were identified and automatically introduced. In this case, these processes were associated with the movements of materials between the various machines, which were identified automatically. From the complete modelled process, the translation into executable language, specifically into a BPEL sheet, was performed as explain in the previous section. This resulted in the automatic discovery of 18 services associated with the 18 subprocesses of the modelled process, in a one to one mapping.

These 18 services totalled 28 parameters, of which only 3 parameters corresponded to the services related to the functional process. 


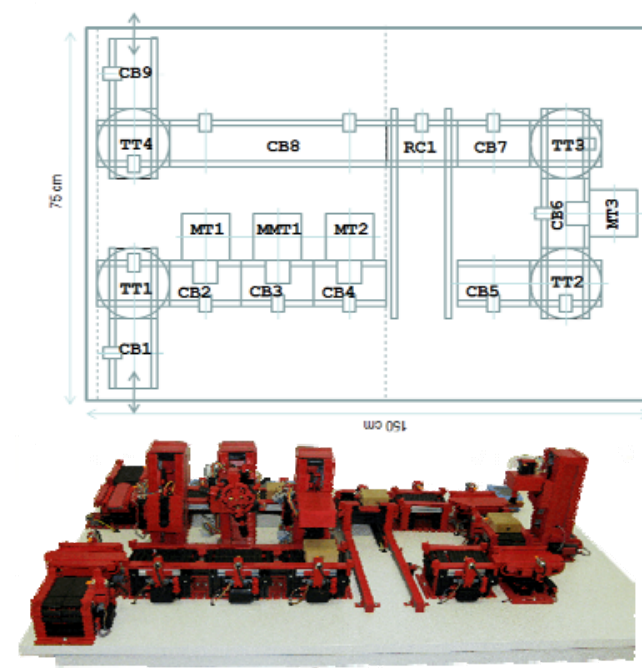

(a)
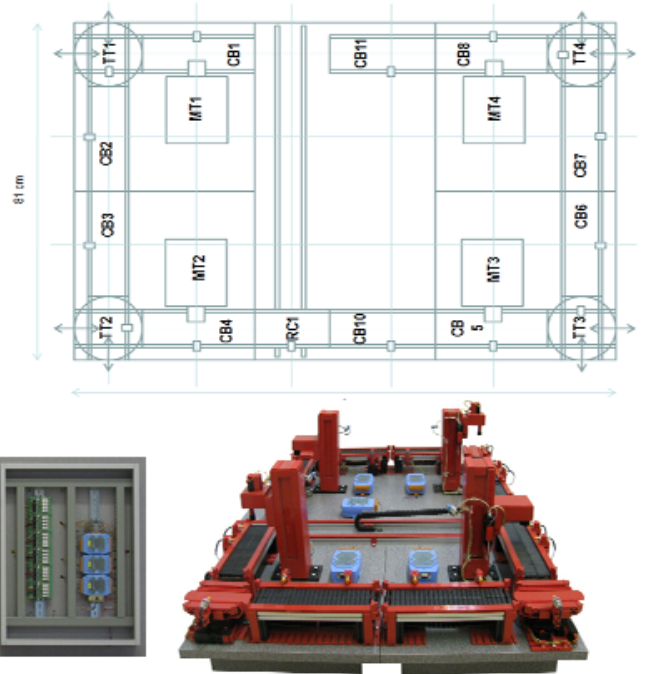

(b)

Figure 5. Comparison between industrial plant 1 (a) and industrial plant 2 (b).

That is, 25 of the parameters involved in the complete process were automatically selected using the specification in the ontology for industrial plant 1 . These parameters were the invocations for 8 services $(8$ parameters) called MoveToEnd offered by the various conveyer belts $(\mathrm{CB}), 2$ services (2 parameters) called TurnMove, offered by turntables (TT), 2 Transport services ( 6 parameters) offered by the rail carriage (RC1), 3 services called MoveToSensor ( 9 parameters) that situate the material in position so it can be processed by the machine tools, 3 services called Process (3 parameters) offered by the machine tools (MT) to perform the transformations on the raw material.

Figure 6 shows the different complexity between the generic functional process model in Figure 6A and the process modelled for the plant where it will be deployed in Figure 6B.

Similarly, the complete process was calculated automatically for plant 2 (Fig. 5b) from the same functional process. In this case, the sub-processes are carried out by other machines: machine MT1 implements the Drilling process; machine MT2 implements the Polishing process and machine MT4 implements the Painting process.

For plant 2, a process composed of 15 sub-processes was obtained, of which 3 were functional and 12 were identified and introduced automatically.

This process modelled in BPMN notation was automatically translated into BPEL language, automatically identifying the 15 services involved in the process.

These 15 services totalled 25 parameters distributed as follows: 6 MoveToEnd services (6 parameters), 1 TurnMove service (1 parameter), 2 Transport services (6 parameters), 3 MoveToSensor services ( 9 parameters) and 3 Process services (3 parameters). Of these parameters, 22 could be calculated from the information contained in the ontology, while the three parameters associated with the Process services had to be provided by the user at compilation time.

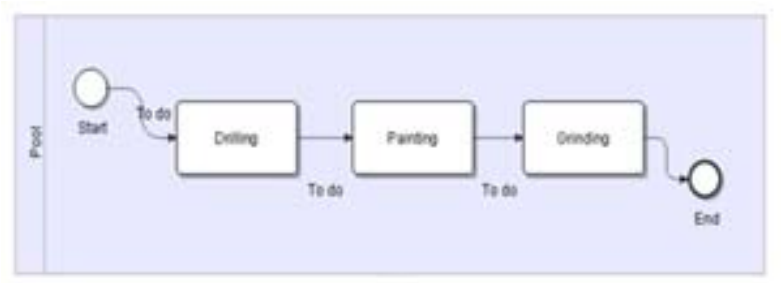

(A)

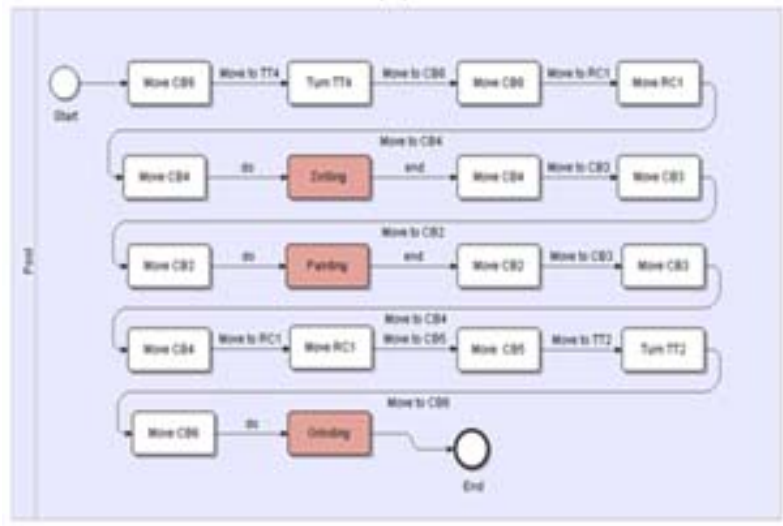

(B)

Figure 6. Comparison between the proposed functional process $(A)$ and the complete process implemented (B) for plant 1.

The tests undertaken show that a high degree of automation in the composition and subsequent translation of the processes modelled with this system was achieved. Specifically, $100 \%$ automation was achieved in the discovery of services, $89.28 \%$ automation in the calculation of the parameters necessary for the invocation of the services for plant 1 and $88 \%$ automation for plant $2,83.3 \%$ automation in 
the composition of the manufacturing processes for plant 1 and $80 \%$ for plant 2 .

These results represent a large reduction in the modelling effort and subsequent deployment of processes as a high level of automation was achieved. This implies a high level of abstraction when performing this effort.

\section{Conclusions}

This paper presents a model and architecture based on SOA for agile manufacturing process modelling independently of the available infrastructure where the models are to be deployed. The proposal is based on the use of ontologies to automatically compose the particular processes that are to be carried out in a given industrial plant from a generic process model and independent of the industrial plant and the available infrastructure where the processes will be finally deployed. The modelled processes were automatically translated to an executable form, achieving close to $90 \%$ automation. Also, because the proposal is based on a SOA architecture implemented via Web Services, the proposed model for process management is independent of the technologies and the hardware of the various industrial plants.

This proposal opens the possibility of modelling more complex and concurrent manufacturing processes. Due to the degree of automation and abstraction achieved from the business level, more complex processes can be tackled, allowing the system to control concurrency over specific resources and to calculate the optimum routes for performing these processes.

Currently, we are working to incorporate into the model a system allowing monitoring and regeneration of manufacturing processes at execution time that will operate automatically, responding to possible failures of the industrial machinery involved in the processes.

\section{References}

[1] C. Younghwan, K. Kwangsoo, K. Cheolhan. "A design chain collaboration framework using reference models". Innternational Journal of Advanced Manufacturing Technology. 26 (1) pp. 183-190. July, 2005.

[2] H. Smith and P. Fingar. "Business Process Management. The Third Wave". Meghan-Kiffer, 2002.

[3] J. F. Chang. "Business Process Management Systems. Strategy and Implementation". Auerbach Publications, 2005.

[4] F. Jammes, H. Smit, J.L. Martinez-Lastra, I.M. Delamer. "Orchestration of Service-Oriented Manufacturing Processes," Proc. of the 10th IEEE International Conference on Emerging Technologies and Factory Automation. Catania, Italy, September 19-22, 2005.

[5] V. Gilart-Iglesias, F. Maciá-Pérez, D. Marcos-Jorquera and F. J.Mora-Gimeno. "Industrial Machines as a Service: Modelling industrial machinery processes".
Proc. of 5th International IEEE Conference on Industrial Informatics (INDIN'07).Vienna, 2007.

[6] F. Macia-Perez; V. Gllart-Iglesias; A. FerrandizColmeiro; J.V Berna-Martinez; J. Gea-Martinez. "New Models of Agile Manufacturing Assisted by Semantic". Proceedings of the IEEE EDOC 2009 workshops and short papers, pp 336-343, Auckland 2009.

[7] Transparent Factory. Manual de usuario y planificación. [Online] Available: http://www.modicon.com, 2001.

[8] U. Topp, P. Müller. "Web based service for embedded devices". International Workshop on Web Service: Research, Standardization and Deployment, 2003.

[9] A.P. Kalogeras, J.V. Gialelis, C.E. Alexakos, M.J. Georgoudakis, S.A. Koubias. "Vertical integration of enterprise industrial systems utilizing Web Service," Proc. of the 5th IEEE International Workshop on Factory Communication System, September 2004.

[10] F. Jammes, H. Smit. "Service-Oriented paradigms in industrial automation," IEEE Transaction on industrial informatics. VOL I. $\mathrm{n}^{\circ}$ 1, pp. 62-70. 2005.

[11] V. Gilart-Iglesias, F. Maciá-Pérez, J.A. Gil-MartínezAbarca and A. Capella-D'alton. "Industrial Machines as a Service: A model based on embedded devices and Web Services". Proc. of 4th International IEEE Conference on Industrial Informatics (INDIN'06). Singapore, 2006.

[12] A. Gomez-Perez, M. Fernandez-Lopez, O. Corcho. “Ontological Engineering".Springer.2004.

[13] A. Siadat, J. Y. Dantan, A. Semenenko, S. Lemaignan. "MASON: A proposal for ontology for Manufacturing Domain". Proc. of the IEEE Workshop on Distributed Intelligent Systems: Collective Intelligence and Its Applications (DIS'06) 2006.

[14] Y. Al-Safi, Valeriy Vyatkin. "An Ontology-Based Reconfiguration Agent for Intelligent Mechatronic Systems". Proc. of the 3rd international conference on Industrial Applications of Holonic and Multi-Agent Systems. Springer-Verlag Berlin Heidelberg 2007.

[15] J. L. Martinez-Lastra, I. M. Delamer. "Semantic Web Services in Factory Automation: Fundamental Insight and Research Roadmap" .IEEE Transactions On Industrial Informatics. vol 2. ${ }^{\circ} 1$. feb 2006.

[16] Y. Yamato, Y. Nakano, H. Sunaga. "Study and Evaluation of Context-Aware Service Composition and Change-Over Using BPEL Engine and Semantic Web Techniques." Proc. of the Fifth IEEE Consumer Communications \& Networking Conference”. 2008.

[17] W. Ren, G. Chen, D. Chen, C. Ping-Low, C. Sun, J. Bing-Zhang, Z. Yang . "Search for Service-Oriented Strategies of Dynamic Composition of Web Services: A Comparative Perspective". 33rd Annual Conference of the IEEE Industrial Electronics Society. 2007.

[18] C. Pedrinaci, C. Brelage, T. Lessen, J. Domingue, D. Karastoyanova, F. Leymann. "Semantic Business Process Management: Scaling up the Management of Business Processes". 2nd IEEE International Conference on Semantic Computing, 2008. 